\title{
Return of test results in Vietnam HIV sentinel surveillance: Implementation and preliminary results
}

\author{
Giang T. Le ${ }^{\star 1}$, Duc H. Bui ${ }^{2}$, Diep T. Vu', Duong C. Thanh ${ }^{4}$, Nghia V. Khuu ${ }^{3}$, \\ Huong T. Phan ${ }^{2}$, Sheryl Lyss ${ }^{1}$ and Abu Abdul-Quader ${ }^{1}$
}

${ }^{1}$ Centers for Disease Control and Prevention, Hanoi, Viet Nam; ${ }^{2}$ Vietnam Authority of HIV/AIDS Control, Ministry of Health, Hanoi, Viet Nam; ${ }^{3}$ Pasteur Institute, Ho Chi Minh, Viet Nam; ${ }^{4}$ National Institute of Hygiene and Epidemiology, Hanoi, Viet Nam

\section{Objective \\ To describe the implementation and preliminary results of returning HIV test results to participants in Vietnam HIV sentinel surveillance.}

\section{Introduction}

Knowledge of one's HIV serostatus helps improve quality of life for those who test positive and decreases the risk of HIV transmission. WHO recommends that all participants in HIV prevalence surveys be provided access to their test results, especially those who test HIV positive [1]. Anonymous Vietnam HIV sentinel surveillance (HSS), implemented since 1994, focuses on people who inject drugs (PWID), female sex workers (FSW), and men who have sex with men (MSM) [2]. According to national guidelines, the HIV testing algorithm for surveillance purposes was based on two tests whereas the diagnostic algorithm for individuals was based on three tests. Thus, surveillance test results could not be returned to participants [3] who were instead encouraged to learn their HIV serostatus by testing at public confirmatory testing sites.

In 2015, a three-test strategy was applied as part of HSS so that test results could be returned to participants.

\section{Methods}

In 2015, return of HIV test results was implemented as a pilot in 16 HSS provinces. HSS participants were asked to identify which of the designated HIV testing and counselling centers (HTC) in the province was most convenient for them. Participants were then given appointment cards with an assigned survey ID to receive their test results at the chosen venue at a specific date and time. Specimens, with assigned survey IDs, were transferred to the respective HIV laboratory at the Province AIDS Center (PAC) for confirmatory testing. The same three-test algorithm was used for surveillance purposes as well as to return confirmatory test results to participants [3]. Final test results were classified as "positive", "negative" or "indeterminate". HIV confirmatory test results were made available at all designated HTC in the provinces within 10 days after blood collection; thus, if a participant presented at a location, date or time that differed from the appointment card, s/he could still receive the test result. In some settings in which provinces integrated HSS with either static or mobile HTC, three rapid tests were used at point-of-care so that same-day test results were available. In this case, participants received test results at the end of the specified time regardless of their infection status.

At the HTC, individuals showed their appointment cards. The IDs were used to identify the correct test results which were then given verbally to participants by HTC counsellors. Test results were not returned by phone or email. Individuals who tested positive were immediately referred to HIV treatment and other available health/ social services in the province.

The proportion of participants who received their test results was calculated for each survey group and province.

\section{Results}

The number of provinces that reported returning of HIV test results in 2015 and 2016 were 14 and 15, respectively. Overall, among 15,530 persons tested through HSS in 2015 and 2016, 7,354 persons returned to receive their test results. The proportion of participants who returned for test results varied by province and survey population (table 1). In some provinces where HSS was integrated with HTC, such as Hai Phong and Dong Thap, $100 \%$ of participants received their test results within a day [4].

\section{Conclusions}

Returning HIV test results to HIV surveillance participants is feasible and beneficial in low-income countries like Vietnam. This enhancement facilitates participants learning their serostatus and contributes toward Vietnam's achievement of HIV control [4]. Based on the pilot experiences, Vietnam Ministry of Health decided to extend test result notifications to all 20 HSS provinces in 2017.

Key factors that contributed to the success of the activity were fast turnaround time, roles and level of commitment of PAC, and coordination between the survey and HTC. The returning rate in HSS 2015 and 2016 are promising but these could be improved further. Better coordination and commitment between the survey and HIV testing service are needed to further increase return rates so that HIVpositive individuals can learn their serostatus and be better linked to care and treatment services.

Table 1: Median and range of proportions, by province, of survey participants who received test results, by survey groups in HSS 2015-2016

\begin{tabular}{|c|c|c|c|c|c|c|}
\hline \multirow{2}{*}{ Year } & \multirow{2}{*}{ Populations } & \multirow{2}{*}{ \# of provinces } & \multicolumn{4}{|c|}{ \% of returning for test results } \\
\cline { 4 - 7 } & & & Median & IQR & Min & Max \\
\hline \multirow{3}{*}{2015} & PWID & 14 & 34.7 & $26.5-45.5$ & 9.6 & 100 \\
\cline { 2 - 7 } & FSW & 14 & 36.2 & $20.0-56.0$ & 5.0 & 100 \\
\cline { 2 - 7 } & MSM & 9 & 40.0 & $18.2-51.8$ & 6.4 & 100 \\
\hline \multirow{3}{*}{2016} & PWID & 15 & 43.5 & $24.4-79.3$ & 1.0 & 100 \\
\cline { 2 - 7 } & FSW & 12 & 64.8 & $28.3-79.8$ & 14.0 & 100 \\
\cline { 2 - 7 } & MSM & 5 & 37.5 & $32.7-61.3$ & 16.7 & 80.7 \\
\hline
\end{tabular}

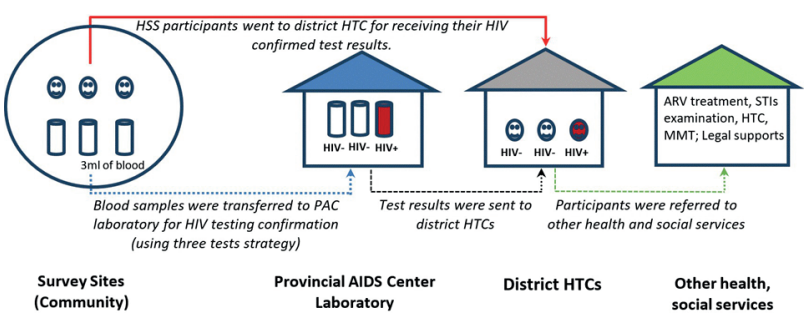

Figure 1: Process of laboratory testing, returning of HIV test results and referral of participants in HSS.

\section{Keywords}

HIV; test return; sentinel surveillance; Vietnam 


\section{ISDS 2018 Conference Abstracts}

\section{Acknowledgments}

The technical assistance provided to Vietnam Government to deploy returning of HIV test results in HSS has been supported by the President's Emergency Plan for AIDS Relief (PEPFAR) thru the CDC.

\section{References}

1. WHO, Guidelines for second generation HIV surveillance: An update: Know your epidemic, 2013.

2. VAAC, Guidance for epidemiological surveillance of HIV/AIDS \& sexually transmitted infections, 2012.

3. MOH, National guideline on HIV serology testing, in Decision 1098/ QD-BYT, 2013.

4. VAAC, Primarily results of HSS, 2016.

*Giang T. Le

E-mail: letonggiang@gmail.com 